\title{
Imaging Findings in MR Imaging-Guided Focused Ultrasound Treatment for Patients with Essential Tremor
}

\author{
M. Wintermark, J. Druzgal, D.S. Huss, M.A. Khaled, S. Monteith, P. Raghavan, T. Huerta, L.C. Schweickert, B. Burkholder, J.J. Loomba, \\ E. Zadicario, Y. Qiao, B. Shah, J. Snell, M. Eames, R. Frysinger, N. Kassell, and W.J. Elias
}

\begin{abstract}
BACKGROUND AND PURPOSE: MR imaging-guided focused sonography surgery is a new stereotactic technique that uses high-intensity focused sonography to heat and ablate tissue. The goal of this study was to describe MR imaging findings pre- and post-ventralis intermedius nucleus lesioning by MR imaging-guided focused sonography as a treatment for essential tremor and to determine whether there was an association between these imaging features and the clinical response to MR imaging-guided focused sonography.
\end{abstract}

MATERIALS AND METHODS: Fifteen patients with medication-refractory essential tremor prospectively gave consent; were enrolled in a single-site, FDA-approved pilot clinical trial; and were treated with transcranial MR imaging-guided focused sonography. MR imaging studies were obtained on a 3T scanner before the procedure and 24 hours, 1 week, 1 month, and 3 months following the procedure.

RESULTS: On T2-weighted imaging, 3 time-dependent concentric zones were seen at the site of the focal spot. The inner 2 zones showed reduced ADC values at 24 hours in all patients except one. Diffusion had pseudonormalized by 1 month in all patients, when the cavity collapsed. Very mild postcontrast enhancement was seen at 24 hours and again at 1 month after MR imaging-guided focused sonography. The total lesion size and clinical response evolved inversely compared with each other (coefficient of correlation $=0.29, P$ value $=.02$ ).

CONCLUSIONS: MR imaging-guided focused sonography can accurately ablate a precisely delineated target, with typical imaging findings seen in the days, weeks, and months following the treatment. Tremor control was optimal early when the lesion size and perilesional edema were maximal and was less later when the perilesional edema had resolved.

ABBREVIATIONS: CRST = Clinical Rating Scale for Tremor; Vim = ventralis intermedius nucleus

$\mathbf{M}_{\mathrm{s}}^{\mathrm{R}}$ $\mathrm{R}$ imaging-guided focused sonography surgery is a new stereotactic technique that uses high-intensity focused sonography to heat and ablate tissue rapidly under closed-loop image guidance and control throughout all steps of the intervention process. MR imaging allows precise intraprocedural localization of the ablation target, verification of safety margins for the sonography treatment, and real-time monitoring of thermal ab-

\section{Received July 5, 2013; accepted after revision August 20.}

From the Departments of Radiology, Neuroradiology Division (M.W., J.D., P.R., T.H., L.C.S., B.B., Y.Q.), Neurosurgery (D.S.H., M.A.K., S.M., J.J.L., R.F., N.K., W.J.E.), and Neurology (B.S.), University of Virginia, Charlottesville, Virginia; Focused Ultrasound Surgery Foundation (J.S., M.E.), Charlottesville, Virginia; and Insightec Ltd (E.Z.), Haifa, Israel.

This work was supported by a grant of the Focused Ultrasound Surgery Foundation (http://www.fusfoundation.org). The content of the article is solely the responsibility of the authors and does not necessarily represent the official views of the Focused Ultrasound Surgery Foundation.

Please address correspondence to Max Wintermark, MD, Department of Radiology, Neuroradiology Division, University of Virginia, PO Box 800170, (FedEx: 1215 Lee St-New Hospital, 1st Floor, Room 1011), Charlottesville, VA 22908-0170; e-mail: Max.Wintermark@virginia.edu

http://dx.doi.org/10.3174/ajnr.A3808 lation dynamics. ${ }^{1-7} \mathrm{MR}$ imaging-guided focused sonography is now accepted in the treatment of soft-tissue disorders, including prostate cancer and uterine fibroids. Intracranial applications for brain tumors ${ }^{8,9}$ and neuropathic pain syndromes ${ }^{10,11}$ are currently under investigation. More recently, MR imaging-guided focused sonography was tested in a clinical trial as a treatment for essential tremor.

Essential tremor is a common and disabling movement disorder with an estimated prevalence of $0.3 \%-5.55 \% .{ }^{12-17}$ Patients with essential tremor may suffer more from the mental effects on quality of life, such as lower perceived health status, ${ }^{18}$ than from actual physical symptoms. ${ }^{19}$ Essential tremor may be medically refractory: up to $30 \%$ of patients do not respond to first-line therapy and may consider surgical options. ${ }^{20}$ Improved imaging and refined electrophysiologic localization have demonstrated that the ventralis intermedius nucleus (Vim) of the thalamus is the most effective target. The ventralis intermedius nucleus was the target for the MR imaging-guided focused sonography treatment in the clinical trial mentioned above.

The goal of this study was to describe findings on MR imaging 
both pre- and post-Vim lesioning by MR imaging-guided focused sonography as a treatment for essential tremor in the 15 patients enrolled in the trial and to determine whether there was an association between these imaging features, the number and/or energy of sonications, and the clinical response to MR imagingguided focused sonography.

\section{MATERIALS AND METHODS \\ Study Population}

Under the auspices of our institutional review board, 15 patients with medication-refractory essential tremor prospectively gave consent; were enrolled in a single-site, FDA-approved pilot clinical trial; and were treated with MR imaging-guided focused sonography targeting the Vim of the thalamus contralateral to the hand-dominant side. A complete medical history was obtained for each patient, along with a detailed physical and neurologic examination. Assessment of the patient's tremor was performed by using a validated Clinical Rating Scale for Tremor (CRST), ${ }^{21}$ which has been shown to be reliable among examiners. ${ }^{22}$ Inclusion and exclusion criteria are reported elsewhere. ${ }^{23}$

\section{Procedure}

The MR imaging-guided focused sonography thalamotomies were performed in a clinical 3T MR imaging system (HD750; GE Healthcare, Milwaukee, Wisconsin) by using a clinical system for focused sonography surgery (ExAblate 4000; InSightec, Haifa, Israel) featuring a hemispheric 1024-element phased array transducer.

The patient's head was fully shaven and then was immobilized within a MR imaging-compatible frame placed by using a local anesthetic. The patient's head rigidly affixed in the frame was carefully positioned in the helmet-like cavity of the sonography transducer. A flexible membrane filled with degassed water for sonography sealed the coupling space between the transducer and head surface. The water circulated at $16^{\circ} \mathrm{C}$ for continuous scalp cooling. The ablation target (ie, the Vim of the thalamus) was localized on 3D T1-weighted MR images by using the anterior/ posterior commissure line as a reference. The Vim of the thalamus was initially targeted with standard indirect measurements from the anterior/posterior commissure line.

The first portion of the procedure consisted of several pretreatment test sonications to confirm that the thermal hot spot was centered in the target location. Several low-power sonications of 10-20 seconds' duration were applied to induce peak temperatures of $<45^{\circ} \mathrm{C}$. These brief temperatures are unlikely to cause lesioning but are easily visualized on MR thermometry images to validate the exact position and size of the hot spot, which typically measures $6 \mathrm{~mm}$ in height and $4 \mathrm{~mm}$ in diameter $(75 \mu \mathrm{L})$.

The treatment itself consisted of several high-power sonications applied in an iterative process guided by MR imaging and MR thermometry. To induce local tissue ablation, we stepwise increased the acoustic power from sonication to sonication to finally achieve a peak temperature between $55^{\circ} \mathrm{C}$ and $60^{\circ} \mathrm{C}$ at the target. Typically, continuous wave sonications of 10-20 seconds duration, up to a maximum acoustic power of $1200 \mathrm{~W}$ and $800 \mathrm{~W}$, respectively, were applied.

Patients were fully awake and responsive during all stages of the intervention. They were monitored and questioned repeatedly to ensure their neurologic integrity and to assess changes in tremor intensity or other sensations experienced during the treatment.

\section{Clinical Outcome Assessment}

All patients were monitored in the hospital for at least 24 hours. Postoperative assessments were performed at 24 hours, 1 week, 1 month, and 3 months and included tremor evaluation, ${ }^{21}$ neurologic examination, and gait testing. ${ }^{24,25}$ The contralateral appendicular tremor was evaluated by using the Clinical Rating Scale for Tremor (having a total of 160 points), which includes 3 parts: 1) observed tremor location and severity, 92 points; 2 ) motor tasks: drawing, handwriting, pouring, 36 points; and 3) disabilities related to speaking, feeding, drinking hygiene, dressing, writing, working, and social activities, 32 points. $^{21}$

\section{Imaging Studies}

Preprocedural imaging included a noncontrast head CT, which was used during the procedure to provide a skull-correction algorithm for transcranial sonication. MR imaging studies were performed on a $3 \mathrm{~T}$ scanner before the procedure and 24 hours, 1 week, 1 month, and 3 months following the procedure. The MR imaging protocol was the same at all 5 time points and included the following sequences: sagittal MPRAGE $(\mathrm{TR}=1900 \mathrm{~ms}, \mathrm{TE}=$ $1.94 \mathrm{~ms}, \mathrm{TI}=900 \mathrm{~ms}$, flip angle $=9^{\circ}, 3 \mathrm{D}, 240$ sections per slab, section thickness $=0.9 \mathrm{~mm}$, distance factor $=50 \%$, base resolution $=256$, phase resolution $=96 \%$, acceleration factor $=2$, whole-brain coverage); axial $\mathrm{T} 1$ spin-echo $(\mathrm{TR}=600 \mathrm{~ms}$, $\mathrm{TE}=$ $8.5 \mathrm{~ms}$, flip angle $=90^{\circ}, 30$ sections, section thickness $=5.0 \mathrm{~mm}$, distance factor $=20 \%$, base resolution $=256$, phase resolution $=$ $100 \%$, whole-brain coverage $)$; axial $\mathrm{T} 2 \mathrm{FSE}(\mathrm{TR}=5410 \mathrm{~ms}, \mathrm{TE}=$ $102 \mathrm{~ms}$, flip angle $=50^{\circ}, 30$ sections, section thickness $=2.0 \mathrm{~mm}$, distance factor $=0 \%$, base resolution $=384$, phase resolution $=$ $85 \%$, acceleration factor $=2$, whole-brain coverage angled with the anterior/posterior commissure line and centered over the thalami); axial FLAIR $(\mathrm{TR}=9000 \mathrm{~ms}, \mathrm{TE}=110 \mathrm{~ms}$, $\mathrm{TI}=2500$ $\mathrm{ms}$, flip angle $=180^{\circ}, 30$ sections, section thickness $=5.0 \mathrm{~mm}$, distance factor $=20 \%$, base resolution $=256$, phase resolution $=$ $100 \%$, whole-brain coverage); axial SWI (TR $=27 \mathrm{~ms}, \mathrm{TE}=20$ $\mathrm{ms}$, flip angle $=15^{\circ}, 3 \mathrm{D}, 104$ sections per slab, section thickness $=$ $1.5 \mathrm{~mm}$, distance factor $=20 \%$, base resolution $=256$, phase resolution $=95 \%$, acceleration factor $=2$, whole-brain coverage); axial and coronal DWI $(\mathrm{TR}=6200 \mathrm{~ms}$, TE $=99 \mathrm{~ms}, 30$ sections, section thickness $=5.0 \mathrm{~mm}$, distance factor $=20 \%$, base resolution $=178$, phase resolution $=100 \%$, acceleration factor $=$ 2, whole-brain coverage); and axial $\mathrm{T} 1$ spin-echo postcontrast $\left(\mathrm{TR}=600 \mathrm{~ms}, \mathrm{TE}=8.5 \mathrm{~ms}\right.$, flip angle $=90^{\circ}, 30$ sections, section thickness $=5.0 \mathrm{~mm}$, distance factor $=20 \%$, base resolution $=$ 256 , phase resolution $=100 \%$, whole-brain coverage).

\section{Data Analysis}

Using a mixed model, we assessed whether the imaging pattern observed after treatment depended on the number of sonications, power used for the last sonication, and maximal temperature during the MR imaging-guided focused sonography treatment. Using correlation analysis, we evaluated whether the imaging fea- 
tures observed after MR imaging-guided focused sonography treatment for essential tremor were associated with the clinical response to the treatment.

\section{RESULTS}

\section{Patients}

Fifteen patients ( 10 men and 5 women; age, $67 \pm 8$ years) were prospectively enrolled in this trial. Twelve were right-handed, and 3, left-handed. The target of the MR imaging-guided focused sonography treatment was the Vim of the thalamus on the contralateral side of the patient's hand dominance.

\section{Procedures}

Patients received an average of 18 sonications (minimum, 11; maximum, 26), with gradual increase in the energy of each sonication. The power used for the last sonication was $845 \pm 243 \mathrm{~W}$ (minimum, $600 \mathrm{~W}$; maximum, $1300 \mathrm{~W}$ ). The duration of the last sonication was between 10 and 16 seconds. The maximal temperature reached during the MR imaging-guided focused sonography treatment was $59 \pm 3^{\circ} \mathrm{C}$ (minimum, $55^{\circ} \mathrm{C}$; maximum, $\left.63^{\circ} \mathrm{C}\right)$.

\section{Clinical Outcome}

Clinical outcome in the 15 patients enrolled in the trial is reported in Table 1.

\section{Imaging Findings}

On T2-weighted imaging, we found a lesion pattern at the site of the focal spot with features similar to those previously found in a

Table 1: Clinical outcome in the 15 patients enrolled in the trial, measured in terms of the Clinical Rating Scale for Tremor ${ }^{\mathrm{a}}$

\begin{tabular}{lcrrc}
\hline \multicolumn{1}{c}{ Time Point } & Mean & SD & Minimum & Maximum \\
\hline Baseline CRST & 54.9 & 14.4 & 38 & 87 \\
24-Hour CRST & 19.3 & 10.8 & 5 & 43 \\
1-Week CRST & 18.5 & 11.0 & 6 & 43 \\
1-Month CRST & 18.7 & 12.0 & 2 & 39 \\
3-Month CRST & 20.3 & 11.0 & 6 & 37 \\
$\begin{array}{c}\text { Outcomes (difference between } \\
\text { baseline and 3-month CRST) }\end{array}$ & 34.6 & 10.4 & 13 & 50 \\
\hline
\end{tabular}

${ }^{a}$ Lower score is favorable; higher score is unfavorable. trial of MR imaging-guided focused sonography treatment of neuropathic pain. ${ }^{10}$ There were 3 concentric zones: a hypointense zone I at the center; a strongly hyperintense zone II demarcated by a hypointense rim; and finally, a fuzzy, slightly hyperintense zone III at the periphery (Fig 1). The diameters of zone I and the thicknesses of zones II and III and their evolution with time are reported in Table 2 and Fig 2.

The focused sonography lesion in the axial plane, defined by the outline of zone II, was round in 10 patients, a vertical (anteroposterior) oval in 2 patients, and a horizontal (left-right) oval in 3 patients. In the 3 patients who required anterior repositioning of the focus, the observed shape was a vertical (anteroposterior) oval in 2 and a horizontal (left-right) oval in 1. The lesion had an elongated oval shape along the $\mathrm{z}$-axis.

A cavity had developed in the location of zones I and II by 24 hours in 13 patients and by 1 week in the remaining 2 patients. This cavity collapsed by 1 month in 12 patients and by 3 months in the remaining 3 patients (Fig 3 ).

The inner 2 zones, zones I and II, showed reduced ADC values $\left(0.52 \pm 0.18 \times 10^{-3} \mathrm{~mm}^{2} / \mathrm{s}\right)$ at 24 hours in all patients except 1 . Diffusion had pseudonormalized by 1 week in 10 patients and by 1 month in the remaining 5 patients, when the cavity collapsed (Fig 3). T1 shortening in the location of the MR imaging-guided focused sonography lesion was present in 9 patients by 1 month and in 1 additional patient by 3 months posttreatment (Fig 3 ). Very mild postcontrast enhancement was seen in 6 patients at 24 hours after the MR imaging-guided focused sonography treatment. Enhancement appeared (or re-appeared) by 1 week in 6

\begin{tabular}{|c|c|c|c|}
\hline T2 Imaging & Zone I & Zone II & Zone III \\
\hline 24 Hours & $2.7 \pm 1.4$ & $2.2 \pm 1.0$ & $3.0 \pm 1.1$ \\
\hline 1 Week & $4.0 \pm 1.6$ & $1.8 \pm 0.8$ & $2.7 \pm 1.8$ \\
\hline 1 Month & $2.1 \pm 1.4$ & $1.2 \pm 0.9$ & $0.1 \pm 0.3$ \\
\hline 3 Months & $0.5 \pm 0.7$ & $0.1 \pm 0.4$ & $0.0 \pm 0.0$ \\
\hline
\end{tabular}
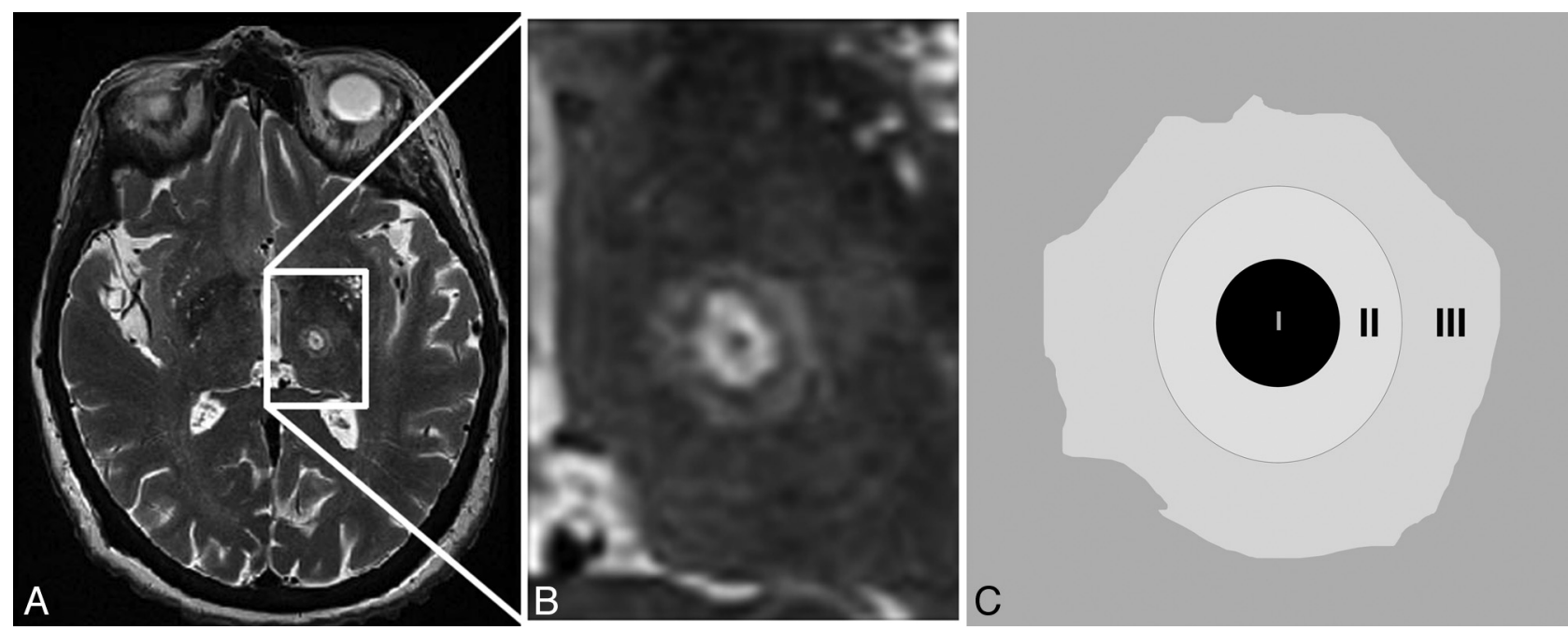

FIG 1. Schematic representation of zones I, II, and III visualized on T2-weighted imaging and the corresponding T2-weighted image of the lesion. On T2-weighted imaging, the patient developed 3 concentric zones at the site of lesioning: a hypointense zone I at the center; a strongly hyperintense zone II demarcated by a hypointense rim; and finally, a fuzzy, slightly hyperintense zone III at the periphery. 


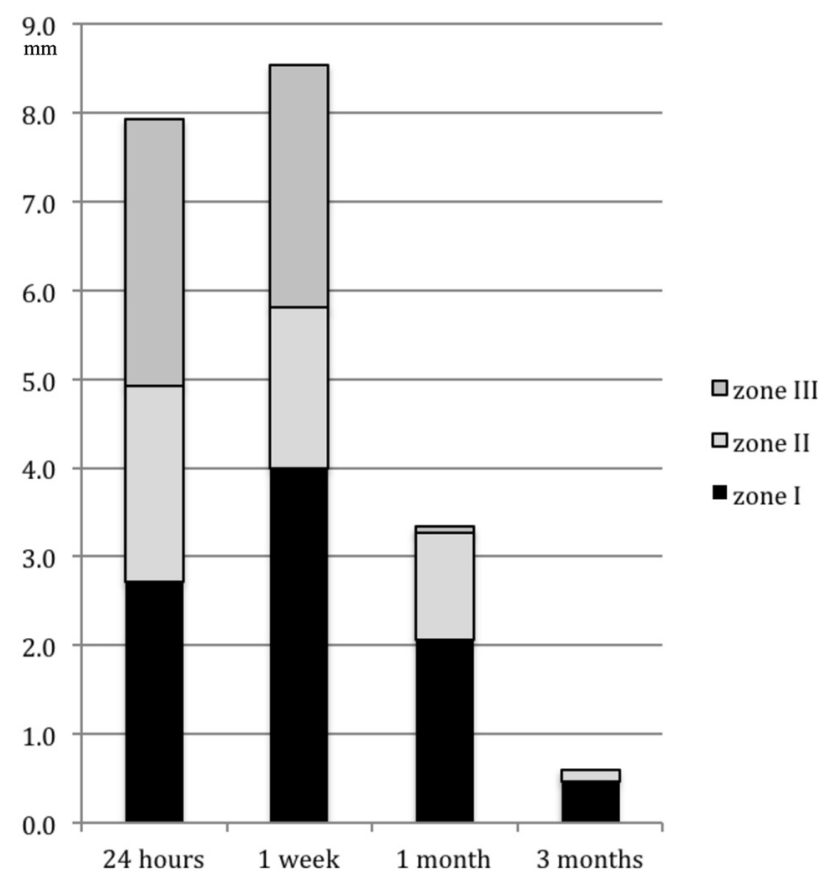

FIG 2. Average diameters of zone I and thicknesses of zones II and III, as seen on T2-weighted imaging, at 24 hours, 1 week, 1 month, and 3 months after the MR imaging-guided focused sonography treatment, all expressed in millimeters.

patients, was present in all patients by 1 month, and had resolved by 3 months in all except 3 patients (Fig 3 ).

No intracerebral hemorrhage occurred, but blood products responsible for artifacts on susceptibility-weighted imaging were seen within zones I and II at all time points (Fig 3).

\section{Relationship between the Imaging Pattern after Treatment and Characteristics of the MR Imaging-Guided Focused Sonography Treatment}

We could not find any significant relationship between the imaging characteristics of the lesions and the number of sonications $(P$ values ranging from .13 to .94$)$, the final power ( $P$ values ranging from .29 to .98 ), or the maximal temperature reached ( $P$ values ranging from .13 to .99).

\section{Relationship between the Imaging Pattern after Treatment and the Clinical Response to the MR Imaging-Guided Focused Sonography Treatment}

We observed that the total lesion size (including zone III) and the clinical response measured based on the CRST score, especially its part B, evolved inversely compared with each other (coefficient of correlation $=0.29, P$ value $=.02)$. Tremor control was optimal at 1 week when the lesion size and the perilesional edema were maximal, and it was less at 1 or 3 months when the perilesional edema had resolved and the total lesion size was smaller. On the other hand, adverse effects were most prominent during the first postoperative week and tended to resolve with the resolution of perilesional edema. Lesion collapse did not mean loss of tremor control. In addition, in the 2 patients who were outliers in terms of tremor control (ie, in the 2 patients who responded less well to the treatment), the imaging characteristics were not significantly different from those in the other patients. More specifically, the total

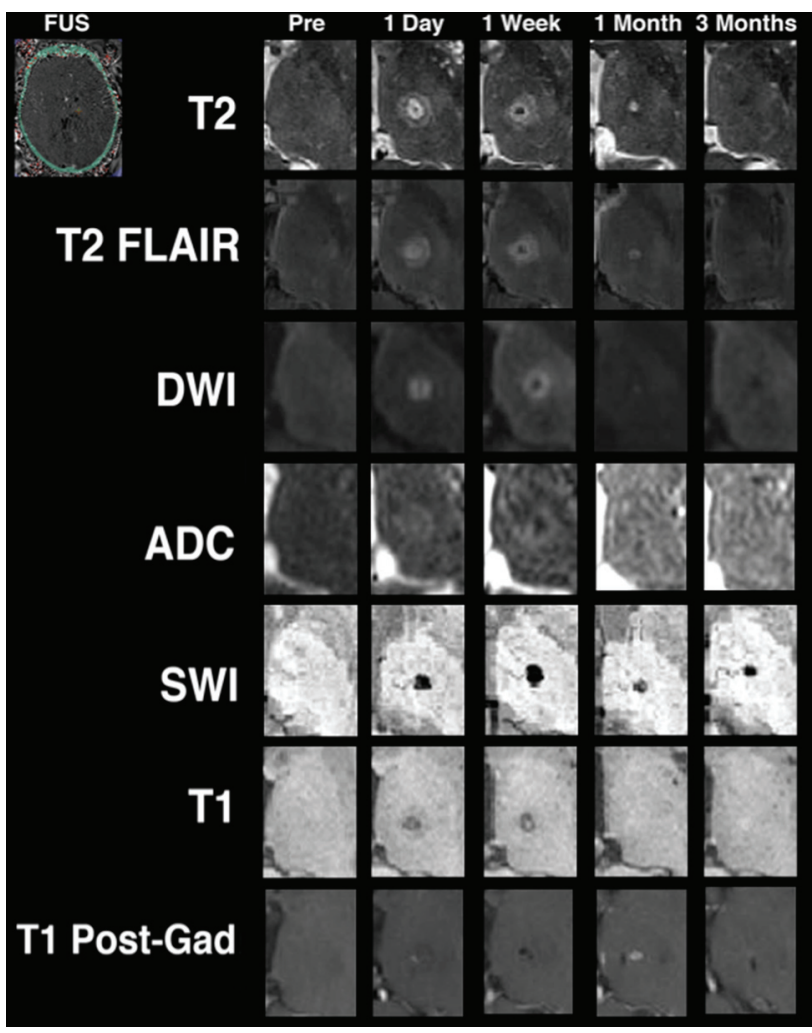

FIG 3. A 76-year-old, right-handed woman with essential tremor treated with MR imaging-guided focused sonography lesioning of the left Vim of the thalamus (thermography image obtained during the treatment is in the left upper corner). On T2-weighted imaging, the patient developed 3 concentric zones at the site of lesioning. Zone II corresponds to vasogenic edema and was seen at 24 hours and 1 week and then resolved. Zones I and II evolved into a round cavity in which diffusion was restricted by 24 hours after treatment. Diffusion pseudonormalized by 1 month when the cavity collapsed. T1 shortening was observed at 1 month and 3 months. Very mild enhancement was seen at 24 hours, likely resulting from the reversible alteration of the blood-brain barrier caused by the focused sonography treatment. Enhancement reappeared by 1 week and peaked at 1 month, by then likely representing neoangiogenesis. No major bleed occurred at any time point, but blood products responsible for susceptibility artifacts were seen within zones I and II at all time points.

lesions were not smaller, and these 2 patients did not show less perilesional edema.

\section{DISCUSSION}

Typical imaging findings following the MR imaging-guided focused sonography treatment for essential tremor consisted of 3 concentric zones at the site of lesioning. These findings were consistent with those previously reported in a trial of MR imagingguided focused sonography lesioning of the contralateral thalamus for neuropathic pain. ${ }^{10}$ Zone III was consistent with vasogenic edema ${ }^{26,27}$; it was typically seen at 24 hours and 1 week and then resolved. This appearance of lesions and their evolution with time was similar to the imaging findings observed for stereotactic radiofrequency thalamotomies but quite different from those observed with gamma knife radiosurgery. ${ }^{28}$ Direct comparison between the different treatment modalities in terms of imaging is available in pigs, ${ }^{29}$ and further studies comparing MR imaging-guided focused sonogra- 
phy with radiofrequency ablation should be conducted in humans.

Zones I and II showed restricted diffusion at 24 hours that pseudonormalized by 1 week or 1 month, when the cavity developing in the location of zones I and II collapsed. Therefore, zones I and II likely represent areas of coagulation necrosis and cytotoxic edema. ${ }^{8,26}$

T1 shortening at the site of lesioning was seen in a little more than half of patients, typically starting at 1 month. Very mild enhancement was seen at 24 hours, likely resulting from the reversible alteration of the blood-brain barrier caused by the focused sonography treatment. ${ }^{30}$ This alteration of the blood-brain barrier typically happens within the first 24 hours and then resolves, ${ }^{31}$ explaining that enhancement was captured at 24 hours in only 6 patients. Enhancement reappeared by 1 week and peaked at 1 month. Further investigation in animal models is needed to elucidate the exact nature of this enhancement, which might be related to neovascularization. ${ }^{32}$ Of note, in the previous application of MR imaging-guided focused sonography for neuropathic pain syndromes, ${ }^{10}$ enhancement was seen at 24 hours and had resolved by 48 hours. Patients were not imaged beyond 48 hours. As such, the 1-month enhancement we are reporting could not be assessed in the neuropathic pain trial.

The focused sonography lesion was round or a horizontal or vertical oval. In patients in whom the focus was repositioned, the lesion was oval; however, the oval shape was also observed in patients for whom the focus was not repositioned.

We found a relationship between the size of the lesion on imaging and the response of the tremor to the treatment, suggesting that tremor control is influenced not only by the lesion size and the amount of perilesional edema but also, most likely, by lesion location.

As a limitation to our study, we acknowledge the limited sample size of our study population. The lack of significance of most of our analyses may reflect the fact that our study was underpowered. Further studies with larger sample size are needed for additional characterization of imaging findings in MR imagingguided focused ultrasound treatment.

\section{CONCLUSIONS}

High-intensity focused sonography can accurately produce controlled heating to a precise intracranial target, with typical imaging findings seen in the days, weeks, and months following the treatment. Tremor control was optimal early when the lesion size and perilesional edema were maximal and was less later when the perilesional edema had resolved and the total lesion size was smaller. Further studies should be conducted to better understand the value of imaging findings either as a guidance for improving the treatment procedure or as a prediction for long-term clinical outcome.

Disclosures: Max Wintermark—RELATED: Grant: Focused Ultrasound Surgery Foundation*; UNRELATED: Board Membership: Bayer, * St. Jude Medical*; Grants/Grants Pending: GE Healthcare, ${ }^{*}$ Philips Healthcare. ${ }^{*}$ Johanna J. Loomba—RELATED: Grant: Focused Ultrasound Surgery Foundation, ${ }^{*}$ Comments: This nonprofit provided institutional grant funding for the ET001 study. Eyal Zadicario is an employee of InSightec Ltd. Neal Kassell is a founding member of the board of the Focused Ultrasound Surgery Foundation, as well as a director and shareholder of InSightec Ltd.
RELATED: Board Membership: InSightec. William Jeff Elias receives research support from the Focused Ultrasound Surgery Foundation for clinical and preclinical studies, * as well as an honorarium for serving on the movement disorder steering committee of the Focused Ultrasound Surgery Foundation. *Money paid to the institution.

\section{REFERENCES}

1. Jolesz FA, McDannold N. Current status and future potential of MRI-guided focused ultrasound surgery. J Magn Reson Imaging 2008;27:391-99

2. Moonen CT, Quesson B, Salomir R, et al. Thermal therapies in interventional MR imaging: focused ultrasound. Neuroimaging Clin N Am 2001;11:737-47

3. Salomir R, Delemazure AS, Palussiere J, et al. Image-based control of the magnetic resonance imaging-guided focused ultrasound thermotherapy. Top Magn Reson Imaging 2006;17:139-51

4. Cline HE, Schenck JF, Hynynen K, et al. MR imaging-guided focused ultrasound surgery. J Comput Assist Tomogr 1992;16:956-65

5. Jolesz FA, Bleier AR, Lauter RS. Laser surgery benefits from guidance by MR. Diagnostic Imaging 1990;12:103-08

6. Jolesz FA, Blumenfeld SM. Interventional use of magnetic resonance imaging. Magn Reson Q 1994;10:85-96

7. Jolesz FA, Moore GJ, Mulkern RV, et al. Response to and control of destructive energy by magnetic resonance. Invest Radiol 1989;24:1024-27

8. Ram Z, Cohen ZR, Harnof S, et al. Magnetic resonance imagingguided, high-intensity focused ultrasound for brain tumor therapy. Neurosurgery 2006;59:949-55, discussion 955-56

9. McDannold N, Clement GT, Black P, et al. Transcranial magnetic resonance imaging-guided focused ultrasound surgery of brain tumors: initial findings in 3 patients. Neurosurgery 2010;66:323-32, discussion 332

10. Martin E, Jeanmonod D, Morel A, et al. High-intensity focused ultrasound for noninvasive functional neurosurgery. Ann Neurol 2009;66:858-61

11. Jeanmonod D, Werner B, Morel A, et al. Transcranial magnetic resonance imaging-guided focused ultrasound: noninvasive central lateral thalamotomy for chronic neuropathic pain. Neurosurg Focus 2012;32:E1

12. Bharucha NE, Bharucha EP, Bharucha AE, et al. Prevalence of essential tremor in the Parsi community of Bombay, India. Arch Neurol 1988;45:907-08

13. Haerer AF, Anderson DW, Schoenberg BS. Prevalence of essential tremor. Results from the Copiah County study. Arch Neurol 1982;39:750-51

14. Hornabrook RW, Nagurney JT. Essential tremor in Papua, New Guinea. Brain 1976;99:659-72

15. Rajput AH, Offord KP, Beard CM, et al. Essential tremor in Rochester, Minnesota: a 45-year study. J Neurol Neurosurg Psychiatry 1984;47:466-70

16. Rautakorpi I, Takala J, Marttila RJ, et al. Essential tremor in a Finnish population. Acta Neurologica Scandinavica 1982;66:58-67

17. Nguyen HV, Ngian V, Cordato D, et al. Quality of life in a random sample of community dwelling older patients with essential tremor. Acta Neurol Scand 2007;116:289-92

18. Woods SP, Scott JC, Fields JA, et al. Executive dysfunction and neuropsychiatric symptoms predict lower health status in essential tremor. Cogn Behav Neurol 2008;21:28-33

19. Lorenz D, Schwieger D, Moises H, et al. Quality of life and personality in essential tremor patients. Mov Disord 2006;21:1114-18

20. Zesiewicz TA, Elble R, Louis ED, et al. Practice parameter: therapies for essential tremor: report of the quality standards subcommittee of the American Academy of Neurology. Neurology 2005;64:2008-20

21. Fahn S, Tolosa E, Marin C. Clinical rating scale for tremor. In: Jankovic J, Tolosa E, eds. Parkinson's Disease and Movement Disorders. Baltimore: Urban \& Schwarzenberg; 1988

22. Stacy MA, Elble RJ, Ondo WG, et al. Assessment of interrater and intrarater reliability of the Fahn-Tolosa-Marin tremor rating scale in essential tremor. Mov Disord 2007;22:833-38 
23. Elias J, Huss D, Voss T, et al. A pilot study of focused ultrasound thalamotomy for essential tremor. N Engl J Med 2013;369:640-48

24. Herman T, Inbar-Borovsky N, Brozgol M, et al. The dynamic gait index in healthy older adults: the role of stair climbing, fear of falling and gender. Gait Posture 2009;29:237-41

25. Wrisley DM, Marchetti GF, Kuharsky DK, et al. Reliability, internal consistency, and validity of data obtained with the functional gait assessment. Phys Ther 2004;84:906-18

26. Chen L, Bouley DM, Harris BT, et al. MRI study of immediate cell viability in focused ultrasound lesions in the rabbit brain. J Magn Reson Imaging 2001;13:23-30

27. Hynynen K, McDannold N, Clement G, et al. Pre-clinical testing of a phased array ultrasound system for MRI-guided noninvasive surgery of the brain: a primate study. Eur J Radiol 2006;59:149-56

28. Tomlinson FH, Jack CR Jr, Kelly PJ. Sequential magnetic resonance imaging following stereotactic radiofrequency ventralis lateralis thalamotomy. J Neurosurg 1991;74:579-84

29. Elias WJ, Khaled M, Hilliard JD, et al. A magnetic resonance imaging, histological, and dose modeling comparison of focused ultrasound, radiofrequency, and gamma knife radiosurgery lesions in swine thalamus. J Neurosurg 2013;119:307-17

30. Hynynen K, McDannold N, Vykhodtseva N, et al. Non-invasive opening of BBB by focused ultrasound. Acta Neurochir Suppl 2003;86:555-58

31. Hynynen K, McDannold N, Vykhodtseva N, et al. Noninvasive MR imaging-guided focal opening of the blood-brain barrier in rabbits. Radiology 2001;220:640-46

32. Smirniotopoulos JG, Murphy FM, Rushing EJ, et al. Patterns of contrast enhancement in the brain and meninges. Radiographics 2007; 27:525-51 